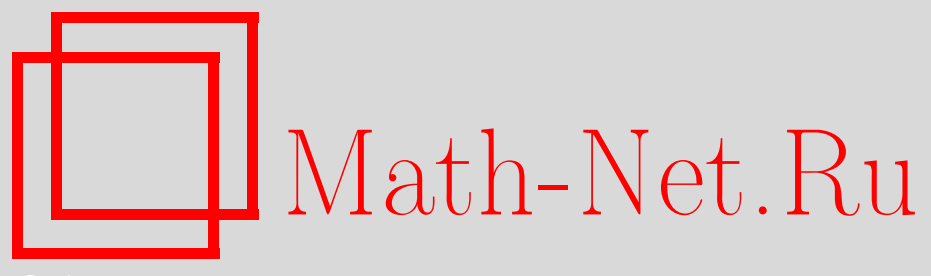

Е. С. Голод, Г. А. Погудин, Модули нулевой горенштейновой размерности над алгебрами графов, Матем. сб., 2016, том 207, номер 7, 81-100

DOI: https://doi.org/10.4213/sm8563

Использование Общероссийского математического портала Math-Net.Ru подразумевает, что вы прочитали и согласны с пользовательским соглашением http://www . mathnet.ru/rus/agreement

Параметры загрузки:

IP : 3.80 .181 .102

26 апреля 2023 г., $17: 57: 25$

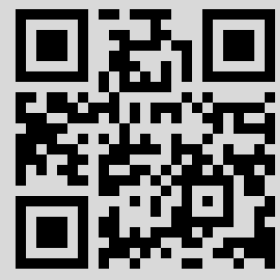




\author{
Е. С. Голод, Г. А. Погудин
}

\title{
Модули нулевой горенштейновой размерности над алгебрами графов
}

\begin{abstract}
Показывается, что несвободные модули нулевой горенштейновой размерности над алгеброй графа существуют в том и только том случае, если граф - дерево. Дается классификация таких модулей.

Библиография: 19 названий.
\end{abstract}

Ключевые слова: горенштейнова размерность, СІ-размерность.

DOI: $10.4213 / \mathrm{sm} 8563$

\section{§ 1. Введение}

Мы рассматриваем нётеровы локальные коммутативные кольца, которые для упрощения ситуации будут предполагаться локальными алгебрами, т.е. содержащими поле вычетов. Все рассматриваемые модули предполагаются конечно порожденными. Для локальных алгебр, представляющих гиперповерхностные особенности, или, более общо, полные пересечения, в ряде работ исследовался вопрос о классификации таких алгебр, имеющих конечный коэн-маколеев тип представления, т.е. конечное число классов изоморфизма неразложимых максимальных модулей Коэна-Маколея (см. [1]).

Для горенштейновых локальных колец класс максимальных модулей Коэна-Маколея совпадает с классом модулей нулевой горенштейновой размерности, называемых также вполне рефлексивными (см. [2], [3]); в другой терминологии понятие горенштейново проективных модулей определено и изучается над произвольными ассоциативными кольцами.

ОПРЕДЕЛЕНИЕ 1. Модуль $M$ над кольцом $R$ имеет нулевую горенштейнову размерность, если он удовлетворяет следующим условиям:

1) естественный гомоморфизм $M \rightarrow M^{* *}$ биективен (здесь через $M^{*}=$ $\operatorname{Hom}_{R}(M, R)$ обозначен сопряженный модуль);

2) $\operatorname{Ext}_{R}^{i}(M, R)=0$ и $\operatorname{Ext}_{R}^{i}\left(M^{*}, R\right)=0$ для всех $i>0$.

ОПРЕДЕЛЕНИЕ 2. Модуль $M$ над кольцом $R$ называется горенштейново проективным, если существует бесконечный в обе стороны ациклический комплекс проективных модулей $\cdots \rightarrow P_{i} \stackrel{\varphi_{i}}{\longrightarrow} P_{i-1} \rightarrow \cdots$ такой, что для некоторого $i$ имеет место изоморфизм $M \cong \operatorname{Ker} \varphi_{i}$ и для всякого проективного модуля $P$ комплекс $\cdots \leftarrow \operatorname{Hom}\left(P_{i}, P\right) \leftarrow \operatorname{Hom}\left(P_{i-1}, P\right) \leftarrow \cdots$ также ацикличен.

В случае конечно порожденных модулей над нётеровым кольцом определения 1 и 2 эквивалентны (см. [4; теорема 4.2.6]). 
ОПРЕДЕЛЕНИЕ 3. Будем говорить, что модуль $M$ над кольцом $R$ имеет горенштейнову размерность не больше $n$ (сокращенно будем называть ее G-размерностью), если существует точная последовательность $0 \rightarrow G_{n} \rightarrow \cdots \rightarrow$ $G_{0} \rightarrow M \rightarrow 0$, где $G_{i}$ - модули горенштейновой размерности 0 .

Если такой последовательности не существует ни для какого $n$, то будем говорить, что модуль имеет бесконечную горенштейнову размерность.

G-размерность, если она конечна, не превосходит глубины локального кольца. Поскольку в настоящей работе речь будет идти о нульмерных локальных кольцах, мы будем говорить только о модулях G-размерности 0.

Модули нулевой горенштейновой размерности над произвольным кольцом Коэна-Маколея являются максимальными модулями Коэна-Маколея, но над негоренштейновыми кольцами Коэна-Маколея модуль нулевой G-размерности не обязательно должен быть максимальным модулем Коэна-Маколея, и поэтому естественно рассматривать вопрос о конечности множества классов изоморфизма неразложимых модулей нулевой горенштейновой размерности над негоренштейновыми локальными кольцами.

Однако в работе Ёсино [5] для некоторых негоренштейновых артиновых колец и, более общо, в работах Такахаси [6]-[8] для негоренштейновых гензелевых колец глубины не больше 2 было показано, что над такими кольцами множество классов изоморфизма неразложимых модулей нулевой горенштейновой размерности конечно, только если всякий такой модуль свободен. Дальнейшие результаты в этом направлении были получены в [2], [3].

В связи с этим представляет интерес вопрос о существовании несвободных модулей нулевой горенштейновой размерности для каких-либо классов негоренштейновых локальных колец. Этот вопрос был подвергнут детальному изучению в упомянутой работе Ёсино [5] для простейшего класса локальных колец - колец с нулевым кубом максимального идеала (там же показано, что для колец с нулевым квадратом максимального идеала таких модулей не существует). В этой работе были получены весьма нетривиальные условия, которым должна удовлетворять алгебра $R$, если над ней существует несвободный модуль нулевой G-размерности (эти условия с некоторыми дополнительными обсуждениями собраны в §2). Также в работе Ёсино показано, что эти условия являются достаточными для существования несвободного $R$-модуля нулевой G-размерности, если $R$ обладает нетривиальной деформацией, т.е. представляется в виде $R=S / f S$, где $(S, \mathfrak{n})$ - одномерная локальная алгебра Коэна-Маколея минимальной кратности (существует неделитель нуля $x \in \mathfrak{n}$, для которого $\left.\mathfrak{n}^{2}=x \mathfrak{n}\right)$ и $f \in \mathfrak{n}^{2}-$ неделитель нуля. В работе Велике [9] приведен пример модуля нулевой G-размерности над алгеброй с нулевым кубом максимального идеала, не имеющей нетривиальных деформаций.

Материал в статье организован следующим образом. В 22 обсуждаются некоторые необходимые условия существования несвободных модулей нулевой горенштейновой размерности над локальными алгебрами с $\mathfrak{m}^{3}=0$. В $\S 3$ обсуждаются циклические модули над такими алгебрами. В $\S 4$ доказывается критерий изоморфизма модулей нулевой горенштейновой размерности над такими алгебрами. В $\S 5-7$ изучаются и классифицируются модули нулевой горенштейновой размерности над алгебрами деревьев. В 88 показывается, что 
большинство алгебр деревьев не имеет нетривиальных деформаций. Более того, над ними так же, как и в примере Велике, не существует несвободных модулей конечной CI-размерности (о CI-размерности см. §8).

\section{§ 2. Необходимые условия существования несвободных модулей нулевой G-размерности над локальной алгеброй с нулевым кубом максимального идеала}

Пусть $(R, \mathfrak{m})$ - локальная алгебра над полем $k=R / \mathfrak{m}$ с $\mathfrak{m}^{2} \neq 0$ и $\mathfrak{m}^{3}=0$. На такой алгебре (см. [5]) можно ввести структуру градуированного кольца $R=$ $R_{0} \oplus R_{1} \oplus R_{2}$, где $R_{0}=k$ и $R_{2}=\mathfrak{m}^{2}$. Если $M$ - модуль нулевой G-размерности, не имеющий свободных прямых слагаемых, то $\mathfrak{m}^{2} M=0$ и на $M$ можно ввести структуру градуированного $R$-модуля $M=M_{0} \oplus M_{1}$, где $M_{1}=\mathfrak{m} M$.

Рассмотрение модулей нулевой горенштейновой размерности как градуированных объектов не влияет на такие их свойства, как изоморфизм и разложимость в прямую сумму, что показывает следующее предложение (наверняка известное, но мы не нашли подходящей ссылки).

ПРеДЛОЖЕНИЕ 1. Пусть $M=\bigoplus_{i \geqslant 0} M_{i} u N=\bigoplus_{i \geqslant 0} N_{i}$ - градуированные модули, порожденные пространствами $M_{0}$ u $N_{0}$ соответственно. Если $M$ и $N$ изоморфны как неградуированные модули, то они также изоморфны как градуированные модули. Если $М$ разлагается в прямую сумму двух ненулевых подмодулей, то М разлагается в прямую сумму двух ненулевых однородных подмодулей.

ДокАзАТЕЛьство. Так как $M$ порождается элементами степени 0, то всякий гомоморфизм $f: M \rightarrow N$ единственным образом разлагается в сумму $f=$ $f_{0}+f_{1}$, где $f_{0}$ - гомоморфизм степени 0 , а $f_{1}$ отображает $M_{i}$ в $N_{i+1} \oplus N_{i+2} \oplus \cdots$. При этом для композиции гомоморфизмов $M \stackrel{f}{\rightarrow} N \stackrel{g}{\rightarrow} P$ выполнено $(g \circ f)_{0}=$ $g_{0} \circ f_{0}$. Поэтому если $f: M \rightarrow N, g: N \rightarrow M-$ взаимно обратные изоморфизмы, то $g_{0} \circ f_{0}=\mathrm{id}_{M}$ и $f_{0} \circ g_{0}=\mathrm{id}_{N}$, т.е. $f_{0}, g_{0}$ - взаимно обратные однородные изоморфизмы.

Если $p: M \rightarrow M-$ идемпотентный гомоморфизм, то $p_{0}^{2}=p_{0}$, причем из $p_{0}=0$ следует $p=0$, так как $p_{1}$ не может быть ненулевым идемпотентом.

Пусть $r=\operatorname{dim}_{k}(0: R \mathfrak{m})-$ размерность цоколя $R$. Если $R$ негоренштейново, то $r>1$. В работе Ёсино получен следующий результат.

Теорема 1 (см. [5; теорема 3.1]). Если $(R, \mathfrak{m}, k)$ - негоренштейнова локальная алгебра $с \mathfrak{m}^{3}=0$ и над ней существует несвободный модуль нулевой $\mathrm{G}$-размерности, то она удовлетворяет следующим условиям:

1) $\left(0::_{R} \mathfrak{m}\right)=\mathfrak{m}^{2}$

2) $\operatorname{dim}_{k} \mathfrak{m} / \mathfrak{m}^{2}=\operatorname{dim}_{k} R_{1}=r+1$;

3) $R$ как градуированная алгебра козюлева, т.е. $R$-модуль $k$ имеет минимальную свободную резольвенту вида

$$
\cdots \rightarrow R^{b_{i}} \stackrel{d_{i}}{\longrightarrow} R^{b_{i-1}} \rightarrow \cdots \rightarrow R_{0} \rightarrow k \rightarrow 0,
$$

в которой все отображения $d_{i}$ задаются матрицами из элементов $R_{1}$ (линейная резольвента) и, в частности, определяющие соотношения алгебры $R$ являются квадратичными формами; 
4) ряд Басса $B_{R}(t)=\sum_{i=0}^{\infty} \mu_{i} t^{i}$, где $\mu_{i}=\operatorname{dim}_{k} \operatorname{Ext}_{R}^{i}(k, R)$, имеет вид

$$
B_{R}(t)=\frac{r-t}{1-r t}
$$

5) если $M-R$-модуль нулевой G-размерности, не содержащий свободных прямых слагаемых, и $b=\operatorname{dim}_{k} M / \mathfrak{m} M$, mо $0:_{M} \mathfrak{m}=\mathfrak{m} M, \operatorname{dim}_{k} \mathfrak{m} M=r b$, $\operatorname{dim}_{k} M^{*} / \mathfrak{m} M^{*}=b$ и $M$ имеет линейную минимальную свободную резольвенmу вида

$$
\cdots \rightarrow R^{b} \stackrel{d_{3}}{\longrightarrow} R^{b} \stackrel{d_{2}}{\longrightarrow} \cdots \stackrel{d_{1}}{\longrightarrow} R^{b} \rightarrow M \rightarrow 0,
$$

причем для всякого $i \geqslant 1$ выполнено $\operatorname{Im} d_{i} \supset \mathfrak{m}^{2} R^{b}$.

ЗАмечАниЕ 1 . Если $(R, \mathfrak{m})$ - артинова локальная алгебра, над которой существует несвободный модуль нулевой G-размерности, то для нее выполнено следующее условие:

$\left(1^{\prime}\right) \mathfrak{m}$ не разлагается в прямую сумму ненулевых идеалов.

Действительно, если $\mathfrak{m}=I \oplus J$ и $M$ - неразложимый несвободный $R$-модуль нулевой горенштейновой размерности, то

$$
M^{*}=\operatorname{Hom}_{R}(M, R)=\operatorname{Hom}_{R}(M, \mathfrak{m})=\operatorname{Hom}_{R}(M, I) \oplus \operatorname{Hom}_{R}(M, J) .
$$

Поскольку $I$ и $J$ имеют ненулевые цоколи, то оба слагаемых в этом разложении ненулевые, и из $M \cong M^{* *}$ следует противоречие с неразложимостью $M$.

ОПРЕДЕлЕниЕ 4. Алгебру, удовлетворяющую условиям теоремы 1 и условию $\left(1^{\prime}\right)$, будем называть алгеброй Ёсино.

Условие 1) в формулировке теоремы 1 следует из $\left(1^{\prime}\right)$, так как если $\left(0:_{R} \mathfrak{m}\right) \nsubseteq$ $\mathfrak{m}^{2}$, то $\mathfrak{m}$ имеет ненулевое слагаемое, изморфное $k$ (порожденное любым элементом из $\left.\left(0:_{R} \mathfrak{m}\right) \backslash \mathfrak{m}^{2}\right)$. Из этого следует, что если $R$ не обязательно артиново, но негоренштейново, и над ним существует несвободный модуль нулевой горенштейновой размерности, то $R$ удовлетворяет условию 1$)$. Из тех же соображений получаем, что если $M-$ модуль нулевой горенштейновой размерности над негоренштейновым кольцом, то $\left(0:_{M} \mathfrak{m}\right) \subseteq \mathfrak{m} M$.

Из утверждения 4) теоремы 1 следует, в частности, что всякий модуль $M$ нулевой G-размерности над алгеброй Ёсино $R$ с минимальным набором из $b$ порождающих изоморфен образу минимального (т.е. $\operatorname{Ker} \varphi \subset \mathfrak{m} R^{b}$ ) однородного степени 1 гомоморфизма $R^{b} \stackrel{\varphi}{\longrightarrow} R^{b}$, удовлетворяющего условиям $\operatorname{dim}(\operatorname{Ker} \varphi)_{1}=b$ $\left(\right.$ где $(\operatorname{Ker} \varphi)_{1}$ - однородная компонента $\operatorname{Ker} \varphi$ степени 1$)$ и $(\operatorname{Im} \varphi)_{2}=R_{2}^{b}$. Заметим, что для любого минимального однородного гомоморфизма степени 1

$$
\operatorname{dim}_{k}(\operatorname{Ker} \varphi)_{1} \geqslant b, \quad(\operatorname{Im} \varphi)_{2} \subseteq R_{2}^{b},
$$

т.е. указанные условия эквивалентны, поскольку в алгебре Ёсино выполнено $\operatorname{dim}_{k} R_{1}^{b}-\operatorname{dim}_{k} R_{2}^{b}=b$. Используя это замечание, получаем следующую лемму.

Лемма 1. Пусть $R$-алгебра Ёсино и комплекс

$$
F: R^{b} \stackrel{\varphi_{n}}{\longrightarrow} R^{b} \rightarrow \cdots \rightarrow R^{b} \stackrel{\varphi_{1}}{\longrightarrow} R^{b}
$$

таков, что все $\varphi_{i}-$ минимальные гомоморфизмы степени 1. Если комплекс $F$ аиикличен $\left(\right.$ m.е. $H_{i}(F)=0$ при $\left.1 \leqslant i \leqslant n-1\right)$, mо $\operatorname{dim}_{k}\left(\operatorname{Ker} \varphi_{i}\right)_{1}=b$ для всех $1 \leqslant i \leqslant n-1$. Если $\operatorname{dim}_{k}\left(\operatorname{Ker} \varphi_{i}\right)_{1}=b$ для всех $1 \leqslant i \leqslant n$, то $F$ ацикличен. 
ДоказАтельство. Так как $\operatorname{dim}_{k}\left(\operatorname{Im} \varphi_{i}\right)_{1}=b$ для всех $1 \leqslant i \leqslant n$, то если $F$ ацикличен, получаем $\operatorname{dim}_{k}\left(\operatorname{Ker} \varphi_{i}\right)_{1}=b$ для всех $1 \leqslant i \leqslant n-1$.

Обратно, пусть $\operatorname{dim}_{k}\left(\operatorname{Ker} \varphi_{i}\right)_{1}=b$ для $1 \leqslant i \leqslant n$. Тогда $\left(\operatorname{Ker} \varphi_{i-1}\right)_{1}=\left(\operatorname{Im} \varphi_{i}\right)_{1}$ и $\left(\operatorname{Im} \varphi_{i}\right)_{2}=R_{2}^{b}$ при $2 \leqslant i \leqslant n$. Так как $\left(\operatorname{Ker} \varphi_{i}\right)_{2} \subseteq R_{2}^{b}$, то $\left(\operatorname{Ker} \varphi_{i-1}\right)_{2}=\left(\operatorname{Im} \varphi_{i}\right)_{2}$ при $2 \leqslant i \leqslant n$.

Сформулируем нужное нам в дальнейшем предложение, которое в случае $M=k$ доказано в [10; теорема 2.11.1], и приведем его простое доказательство для произвольного модуля $M$. Пусть $R=k \oplus R_{1} \oplus \cdots$ - стандартная градуированная алгебра, а $M=\bigoplus_{i \geqslant 0} M_{i}-$ конечно порожденный градуированный $R$-модуль. Обозначим через $h_{i}(M)=\operatorname{dim}_{k} M$ размерности градуированных компонент и через $H_{M}(t)=\sum_{i=0}^{\infty} h_{i}(M) t^{i}-$ ряд Гильберта модуля $M$. Пусть $F: \cdots \rightarrow F_{1} \rightarrow F_{0} \rightarrow M \rightarrow 0$ - минимальная свободная резольвента $M$, где $F_{i}=\bigoplus_{j \geqslant i} R(-j)^{b_{i j}}$. Неотрицательные целые числа $b_{i j}$ называются градуированными числами Бетти модуля $M$, числа $b_{i}=\sum_{j \geqslant i} b_{i j}$ называются числами Бетти. Через $P_{M}(t)=\sum_{i=0}^{\infty} b_{i} t^{i}$ обозначим ряд Пуанкаре для $M$. Говорят, что модуль $M$ имеет линейную резольвенту, если $b_{i j}=0$ при $j>i$. Тогда верно следующее утверждение.

ПРЕДЛОЖЕНИЕ 2. Если выполнено соотношение $H_{M}(t)=H_{R}(t) P_{M}(-t)$, mо $M$ имеет линейную резольвенту.

ДокАзАтЕльство. Если выполняется данное соотношение, то для всех $i \geqslant 0$ выполнено рекуррентное соотношение $h_{i}(M)=\sum_{k=0}^{i}(-1)^{k} h_{i-k}(R) b_{k}$. Пусть резольвента для модуля $M$ не является линейной и $i$ - наименьший индекс, где линейность нарушается, т.е. $b_{k j}=0$ при $j>k$ и $i>k$, но $b_{i j} \neq 0$ для некоторого $j>i$. Для этого $i$ помимо выписанного рекуррентного соотношения должно выполняться также соотношение

$$
h_{i}(M)=(-1)^{i} b_{i i}+\sum_{k=0}^{i-1}(-1)^{k} h_{i-k}(R) b_{k},
$$

следующее из точности градуированного комплекса. Так как $b_{i} \neq b_{i i}$, эти равенства не могут выполняться одновременно.

ЗАмечАниЕ 2. Пусть $R=\bigoplus_{i=0}^{s} R_{i}$ - артинова градуированная локальная алгебра над полем $k, R_{0}=k, R_{s} \neq(0)$. Тогда инъективная оболочка поля вычетов $E(k)$ может быть наделена градуировкой $E(k)=\bigoplus_{i=0}^{s} E_{i}$, где $E_{0} \neq 0$ и $\operatorname{dim}_{k} E_{s}=1$, и ряд Басса $B_{R}(t)$ совпадает с рядом Пуанкаре $P_{E(k)}(t)$. В условиях теоремы Ёсино вид ряда Басса $B_{R}(t)$ показывает, что

$$
H_{R}(t) P_{E(k)}(-t)=H_{E(k)}(t),
$$

где через $H$ обозначен соответствующий ряд Гильберта. Отсюда в силу предложения 2 следует, что градуированный $R$-модуль $E(k)$ имеет линейную свободную резольвенту.

Кроме того, в условиях теоремы Ёсино первый модуль сизигий для $E(k)$, также имеющий после сдвига градуировки линейную резольвенту, имеет только две ненулевые однородные компоненты. Если над градуированной алгеброй $R$ существует градуированный модуль $M=M_{1} \oplus M_{2}$, имеющий линейную 
резольвенту, то алгебра $R$ козюлева. Доказательство этого утверждения по существу идентично доказательству козюлевости алгебры в теореме Ёсино, где в качестве $M$ используется модуль нулевой G-размерности, не имеющий свободных прямых слагаемых. Таким образом, условие 4) в теореме 1 следует из условия 3) непосредственно и не требует наличия несвободного модуля нулевой горенштейновой размерности.

ЗАмЕчАниЕ 3. Приведенное выше условие (1') также следует из условия 3) теоремы 1. Более того, имеет место следующее утверждение.

Пусть цоколь градуированной алгебры $R=\bigoplus_{i=0}^{s} R_{i}, s \geqslant 2$, порожденной $R_{1}$, совпадает с $R_{s}$ (тогда $E(k)$ порождается в степени 0 ). Если максимальный однородный идеал $R_{+}$разлагается в прямую сумму двух ненулевых однородных идеалов $R_{+}=R_{+}^{\prime} \oplus R_{+}^{\prime \prime}$, то модуль $E(k)$ не является линейно представимым (т.е. его первый модуль сизигий не порождается линейными соотношениями) и, следовательно, не имеет линейной резольвенты.

Действительно, разложимость в прямую сумму максимального идеала в $R$ равносильна разложимости подмодуля $E(k) / \varepsilon$, где $\varepsilon$ - одномерный цоколь $E(k)$. Согласно предложению 1 модуль $E(k) / \varepsilon$ разлагается в прямую сумму однородных подмодулей $\bar{M}=M_{0} \oplus M_{1}$ и $\bar{N}=N_{0} \oplus N_{1}$. Если $f_{1}, \ldots, f_{p}$ и $g_{1}, \ldots, g_{q}-$ базисы в $M_{0}$ и $N_{0}$ соответственно, то $\left\{f_{1}, \ldots, f_{p}, g_{1}, \ldots, g_{q}\right\}$ можно отождествить с минимальной системой порождающих в $E(k)$. Так как $M_{1} \cap N_{1}=0$, то все линейные соотношения между ними порождаются линейными соотношениями между $f_{1}, \ldots, f_{p}$ и $g_{1}, \ldots, g_{q}$. Подмодули $M=\left\langle f_{1}, \ldots, f_{p}\right\rangle$ и $N=\left\langle g_{1}, \ldots, g_{q}\right\rangle$ в $E(k)$ содержат $\varepsilon$, а поэтому имеется соотношение степени 2 вида

$$
\left(a_{1} f_{1}+\cdots+a_{p} f_{p}\right)+\left(b_{1} g_{1}+\cdots+b_{q} g_{q}\right)=0,
$$

где $a_{i}, b_{j} \in R_{2}$ и заключенные в скобки слагаемые не равны нулю. Однако в любом соотношении, порожденном линейными, такого вида слагаемые должны обращаться в нуль.

ЗАмечание 4 . Пусть $R$ - локальное кольцо и $\operatorname{depth} R=0$. Если $M \rightarrow M^{* *}$ инъективно и модуль $M^{*}$ рефлексивен, то $M$ рефлексивен. Действительно, пусть $M \stackrel{\varphi}{\rightarrow} M^{* *} \rightarrow N \rightarrow 0$; тогда $0 \rightarrow N^{*} \rightarrow M^{* * *} \stackrel{\varphi^{*}}{\longrightarrow} M^{*}$. Но $\varphi^{*}-$ биекция, а значит, $N^{*}=0$. Так как $R$ имеет цоколь, $N=0$.

Без предположения $\operatorname{depth} R=0$ это, вообще говоря, неверно. Пусть $M=$ $(x, y) \subset k[x, y]_{(x, y)}=R$. Тогда $M^{*} \cong R$, но $M$ не рефлексивен.

Покажем теперь, что из G-dim $M^{*}=0$ следует G-dim $M=0$. Пусть $\varphi$ : $M \rightarrow M^{* *}$. Обозначим $\operatorname{Im} \varphi$ через $\bar{M}$. Тогда $\bar{M}^{*}=M^{*}$, а значит, согласно предыдущему, G-dim $\bar{M}=0$. Пусть $N=\operatorname{Ker} \varphi$; тогда $0 \rightarrow N \rightarrow M \rightarrow \bar{M} \rightarrow 0$, откуда

$$
0 \rightarrow \bar{M}^{*} \rightarrow M^{*} \rightarrow N^{*} \rightarrow \operatorname{Ext}_{R}^{1}(\bar{M}, R)
$$

Так как $\operatorname{Ext}_{R}^{1}(\bar{M}, R)=0$ и гомоморфизм между $\bar{M}^{*}$ и $M^{*}$ является биекцией, то $N^{*}=0$, а значит, и $N=0$. 


\section{§ 3. Циклические модули нулевой G-размерности над локальной алгеброй $\mathrm{c} \mathrm{m}^{3}=0$}

ПреДЛОЖеНИЕ 3. Пусть $R=R_{0} \oplus R_{1} \oplus R_{2}$ - стандартная градуированная алгебра с $\operatorname{dim}_{k} R_{2}=\operatorname{dim}_{k} R_{1}-1=r$. Над $R$ существует несвободный ииклический модуль нулевой G-размерности в том и толъко том случае, если существуют такие элементы $p, q \in R_{1}$, что $\operatorname{Ann}_{R} p=(q) u \operatorname{Ann}_{R} q=(p)$.

ДокАЗАтЕЛьСтво. Пусть $M=R / I$ - модуль нулевой G-размерности, $I \neq 0$. Так как $\mathfrak{m}^{2} M=0$, то $\mathfrak{m}^{2} R=R_{2} \subseteq I$. Согласно Ёсино, $\mathfrak{m} M=\mathfrak{m} / I$ имеет размерность $r$. Следовательно, $\operatorname{dim}_{k} I / \mathfrak{m}^{2}=1$. Так как $I$ также имеет нулевую G-размерность и $\operatorname{soc} I=\mathfrak{m}^{2}$, то $\mathfrak{m} I=\mathfrak{m}^{2}$ и $I$ является циклическим, т.е. $I=(p)$ и $\operatorname{dim}_{k} I=r+1$. Пусть

$$
J=\operatorname{Ann}_{R} p=\operatorname{Ann}_{R} I
$$

Тогда $M^{*} \cong J$ - модуль нулевой $\mathrm{G}$-размерности, $\operatorname{soc} J=\mathfrak{m}^{2}$. Согласно Ёсино, так как $\operatorname{dim}_{k} \mathfrak{m}^{2}=\operatorname{dim}_{k} \mathfrak{m} J=r$, то $\operatorname{dim}_{k} J / \mathfrak{m} J=1, J=(q)$ и $\operatorname{dim}_{k} J=r+1$. Модуль $R / J$ имеет нулевую G-размерность, а значит, $I \subseteq \operatorname{Ann}_{R} J$ и $\operatorname{dim}_{k} \operatorname{Ann}_{R} J=$ $r+1$, т.е. $I=\operatorname{Ann}_{R} J$.

Пусть существуют элементы $p$ и $q$, удовлетворяющие условию предложения 3. Несвободность модулей $R /(p)$ и $R /(q)$ очевидна. Заметим, что из-за условия на аннуляторы следует, что ядром умножения на $p$ как отображения $R \rightarrow R$ является в точности $(q)$. Отсюда следует, что $(R /(p))^{*} \cong(q) \cong R /(p)$. Это соотношение сразу дает нам первую часть определения 1 модулей нулевой G-размерности.

Также мы можем построить резольвенту $\cdots \stackrel{q}{\rightarrow} R \stackrel{p}{\rightarrow} R \rightarrow R /(p) \rightarrow 0$, которая при сопряжении перейдет в $0 \rightarrow(q) \rightarrow R \stackrel{p}{\rightarrow} R \stackrel{q}{\rightarrow} \cdots$, т.е. $\operatorname{Ext}_{R}^{i}(R /(p), R)=0$ при $i \geqslant 1$. Оба этих комплекса ацикличны, что и дает нам вторую часть определения 1 модулей нулевой G-размерности.

Замечание 5. Такие $p$ и $q$, что $\operatorname{Ann}_{R}(p)=(q)$ и $\operatorname{Ann}_{R}(q)=(p)$, называются точными делителями нуля. В работе [11] с помощью точных делителей нуля строится целое бесконечное семейство попарно неизоморфных модулей нулевой G-размерности. Отметим, что все модули, построенные в [11], имеют периодическую минимальную резульвенту с периодом 2.

Дальнейшее развитие эта техника получила в работах [12]--[14].

\section{§ 4. Изоморфизм подмодулей}

Лемма 2. Пусть $R$ - локальное кольцо с максимальным идеалом $\mathfrak{m}, F$ свободный $R$-модуль ранга $n u \varphi: F \rightarrow M$ - сюрвективный гомоморфизм $R$-модулей. Если элементы $a_{1}, \ldots, a_{n}$ порождают $M$, то существует базис $e_{1}, \ldots, e_{n}$ свободного модуля $F$ такой, что $\varphi\left(e_{i}\right)=a_{i}$ для всех $i=1, \ldots, n$.

ДокАЗАтельство. Рассмотрим сначала случай, когда $R$ - поле. Пусть $a_{1}, \ldots, a_{k}$ образуют базис $R$-векторного пространства $M$ и $a_{j}=\sum_{i=1}^{k} \lambda_{j i} a_{i}$ для 
всех $j>k$. Выберем $e_{1}, \ldots, e_{k}$ так, что $\varphi\left(e_{i}\right)=a_{i}$, и пусть $e_{k+1}^{\prime}, \ldots, e_{n}^{\prime}-$ некоторый базис $\operatorname{Ker} \varphi$. Положим для всякого $j>k$

$$
e_{j}=e_{j}^{\prime}+\sum_{i=1}^{k} \lambda_{j i} e_{i} .
$$

Тогда $e_{1}, \ldots, e_{n}-$ искомый базис для $F$.

Пусть теперь $R$ - произвольное локальное кольцо, $\bar{\varphi}: F / \mathfrak{m} F \rightarrow M / \mathfrak{m} M-$ индуцированный гомоморфизм и $\bar{a}_{1}, \ldots, \bar{a}_{n}$ - образы $a_{1}, \ldots, a_{n}$ в $M / \mathfrak{m} M$. В силу уже доказанного для полей найдется базис $\bar{e}_{1}, \ldots, \bar{e}_{n}$ в $F / \mathfrak{m} F$, для которого $\bar{\varphi}\left(\bar{e}_{i}\right)=\bar{a}_{i}$. Для базиса $e_{1}^{\prime}, \ldots, e_{n}^{\prime}$, составленного из некоторых представителей классов $\bar{e}_{1}, \ldots, \bar{e}_{n}$, имеем $\varphi\left(e_{i}^{\prime}\right)-a_{i} \in \mathfrak{m} M$ для всех $i=1, \ldots, n$. Так как $\varphi$ отображает $\mathfrak{m} F$ сюръективно на $\mathfrak{m} M$, то можно выбрать такие $u_{i} \in \mathfrak{m} F$, что $\varphi\left(u_{i}\right)=\varphi\left(e_{i}^{\prime}\right)-a_{i}$. Тогда положим $e_{i}=e_{i}^{\prime}-u_{i}$ для $i=1, \ldots, n$. Набор $e_{1}, \ldots, e_{n}$ и будет искомым базисом в $F$.

Лемма 3. Пусть $R$ - либо стандартная градуированная алгебра над полем $k$, либо локальная алгебра с полем вычетов $k, a \mathfrak{m}$ - максимальный однородный или максимальный идеал соответственно. Пусть заданы гомоморфизмы (в градуированном случае - однородные некоторых степеней) конечно порожденных свободных $R$-модулей $F_{2} \underset{\psi_{2}}{\stackrel{\varphi_{2}}{\rightleftarrows}} F_{1} \underset{\psi_{1}}{\stackrel{\varphi_{1}}{\rightleftarrows}} F_{0}$ такие, ито

$$
\operatorname{Ext}_{R}^{1}\left(\operatorname{Coker} \psi_{1}, R\right)=\operatorname{Ext}_{R}^{1}\left(\operatorname{Coker} \varphi_{1}, R\right)=0 .
$$

Тогда $\operatorname{Im} \varphi_{1} \cong \operatorname{Im} \psi_{1}$ в том и только том случае, когда существуют автоморфизмы $\alpha: F_{0} \rightarrow F_{0}$ и $\beta: F_{1} \rightarrow F_{1}$ такие, что $\psi_{1}=\alpha \varphi_{1} \beta$.

ДокАЗАтельство. Заметим, что комплексы $F_{2} \stackrel{\varphi_{2}}{\longrightarrow} F_{1} \stackrel{\varphi_{1}}{\longrightarrow} F_{0} \rightarrow$ Coker $\varphi_{1}$ и $F_{2} \stackrel{\psi_{2}}{\longrightarrow} F_{1} \stackrel{\psi_{1}}{\longrightarrow} F_{0} \rightarrow$ Coker $\psi_{1}$ можно рассматривать как начальные отрезки свободных резольвент для Coker $\varphi_{1}$ и Coker $\psi_{1}$ соответственно. Условие

$$
\operatorname{Ext}_{R}^{1}\left(\operatorname{Coker} \varphi_{1}, R\right)=\operatorname{Ext}_{R}^{1}\left(\operatorname{Coker} \psi_{1}, R\right)=0
$$

равносильно тому, что сопряженные комплексы точны в члене $F_{1}^{*}$, а значит, и просто точны.

Если $\psi_{1}=\alpha \varphi_{1} \beta$, то $\alpha$ изоморфно отображает $\operatorname{Im} \varphi_{1}$ на $\operatorname{Im} \psi_{1}$.

Обратно, пусть $f: \operatorname{Im} \varphi_{1} \rightarrow \operatorname{Im} \psi_{1}$ - изоморфизм, в силу предложения 1 в градуированном случае его можно считать однородным. Обозначим $r_{i}=\operatorname{rank} F_{i}$ для $i=0,1,2$. Выберем в $\operatorname{Im} \varphi_{1}$ систему образующих $a_{1}, \ldots, a_{r_{1}}$. Согласно лемме 3 в $F_{1}$ найдутся такие базисы $e_{1}^{1}, \ldots, e_{r_{1}}^{1}$ и $e_{1}^{2}, \ldots, e_{r_{1}}^{2}$, что $\varphi_{1}\left(e_{i}^{1}\right)=a_{i}$ и $\psi_{1}\left(e_{i}^{2}\right)=f\left(a_{i}\right)$. Поэтому автоморфизм $\widetilde{\beta}: F_{1} \rightarrow F_{1}$, заданный формулой $\widetilde{\beta}\left(e_{i}^{2}\right)=e_{i}^{1}$, удовлетворяет равенству $f \circ \varphi_{1} \circ \widetilde{\beta}=\psi_{1}$.

Тогда $\operatorname{Ker}\left(\varphi_{1} \circ \widetilde{\beta}\right)=\operatorname{Ker} \psi_{1}$, а значит, $\widetilde{\beta}$ определяет изоморфизм $\operatorname{Ker} \varphi_{1}$ с Ker $\psi_{1}$, а тогда в силу точности и $\operatorname{Im} \varphi_{2}$ с $\operatorname{Im} \psi_{2}$. Как и выше, существует автоморфизм $\gamma: F_{2} \rightarrow F_{2}$ такой, что $\widetilde{\beta} \circ \psi_{2} \circ \gamma=\varphi_{2}$.

Переходим к сопряженным отображениям. Так как $\widetilde{\gamma}^{*}-$ изоморфизм, образы $\varphi_{2}^{*}$ и $\psi_{2}^{*}$ изоморфны. Так же, как для отображения $f$, существуют автоморфизмы $\alpha^{*}$ и $\beta^{*}$ для $F_{0}^{*}$ и $F_{1}^{*}$ такие, что $\psi_{1}^{*}=\beta^{*} \circ \varphi_{1}^{*} \circ \alpha^{*}$. Переходя к сопряженным отображениям, получаем требуемое. 
СлеДСтвиЕ 1. В условиях леммы 3 в градуированном случае отображения $\varphi_{1}$ и $\psi_{1}$ имеют одну и ту же степень.

СлеДСтвие 2. Пусть $\operatorname{depth} R=0$ и $A_{1}, A_{2}$ - две матриць над $R$ размеpa $b \times t$ с однородными элементами одной степени в градуированном случае. Пусть подмодули нулевой G-размерности $M_{1}$ и $M_{2}$ в $R^{b}$ порождаются столбцами $A_{1}$ и $A_{2}$ соответственно. Тогда $M_{1} \cong M_{2}$, если и только если существуют обратимые $(b \times b)-u(t \times t)$-матриць $U$ u $V$ такие, что $A_{2}=U A_{1} V$.

ДокаЗАТЕЛЬСтво. Пусть $M_{1}$ и $M_{2}$ изоморфны. Рассмотрим гомоморфизмы $\varphi_{1}$ и $\psi_{1}$ из $R^{t}$ в $R^{b}$, задаваемые матрицами $A_{1}$ и $A_{2}$. Так как $\operatorname{Im} \varphi_{1} \cong$ $\operatorname{Im} \psi_{1}$, то имеет место изоморфизм $\operatorname{Ker} \varphi_{1} \cong \operatorname{Ker} \psi_{1}$. Пусть $R^{s} \stackrel{\varphi_{2}}{\longrightarrow} \operatorname{Ker} \varphi_{1}$ и $R^{s} \stackrel{\psi_{2}}{\longrightarrow} \operatorname{Ker} \psi_{1}$ определяются минимальными системами порождающих в $\operatorname{Ker} \varphi_{1}$ и $\operatorname{Ker} \psi_{1}$ соответственно. Так как $M_{i}$ имеют нулевую G-размерность, то модули $N_{i}=R^{b} / M_{i}$ имеют нулевую G-размерность, откуда

$$
\operatorname{Ext}_{R}^{1}\left(\operatorname{Coker} \varphi_{1}, R\right)=\operatorname{Ext}_{R}^{1}\left(\text { Coker } \psi_{1}, R\right)=0 .
$$

Таким образом, мы попали в условия леммы 3 . Искомые матрицы $U$ и $V$ будут матрицами полученных автоморфизмов $\alpha$ и $\beta$.

\section{§ 5. Модули нулевой G-размерности над алгебрами графов}

Теперь рассмотрим некоторый класс алгебр, для которых будут описаны все модули нулевой G-размерности. Фиксируем основное поле $k$. Рассмотрим граф $G$ на $n$ вершинах, занумерованных числами от 1 до $n$. То, что ребро между вершинами $i$ и $j$ принадлежит графу $G$, будем записывать как $(i, j) \in G$.

ОПРЕДЕЛЕНИЕ 5. Определим алгебру графа $G$ следующим образом:

$$
\begin{aligned}
A_{k}(G) & =k\left[x_{1}, \ldots, x_{n}\right] /\left(\left(x_{1}^{2}, \ldots, x_{n}^{2}, x_{i} x_{j} \mid(i, j) \notin G\right)+\left(x_{1}, \ldots, x_{n}\right)^{3}\right) \\
& \cong k \oplus\left\langle x_{1}, \ldots, x_{n}\right\rangle \oplus\left\langle x_{i} x_{j} \mid(i, j) \in G\right\rangle .
\end{aligned}
$$

Отметим, что алгебра графа получается из алгебры Стенли-Райснера для графа $G$ (см., например, [15]), рассмотренного как симплициальный комплекс, путем факторизации по квадратам образующих.

Особый интерес для нас будет представлять случай, когда $G$ - дерево.

Лемма 4. Пусть $G$ - граф на $n$ вершинах. Если алгебра $A_{k}(G)$ является алгеброй Ёсино, то $G$ - дерево.

ДоказАтельство. Пусть граф $G$ несвязен, т.е. представим в виде объединения $G_{0} \cup G_{1}$ своих подграфов $G_{0}$ и $G_{1}$. Тогда максимальный идеал $A_{k}(G)$ раскладывается в прямую сумму идеалов, каждый из которых порожден переменными, соответствующими вершинам $G_{0}$ и $G_{1}$. Если в $G$ больше $n-1$ ребер, то $A_{k}(G)$ не удовлетворяет условию 2$)$ теоремы 1 . Таким образом, $G$ является деревом.

Звездой будем называть дерево, в котором существует вершина, соединенная со всеми остальными. 
Далее фиксируем дерево $T$ с $n>1$ и будем обозначать $A_{k}(T)$ через $R$. От алгебры многочленов алгебра $R$ наследует естественную градуировку

$$
R=R_{0} \oplus R_{1} \oplus R_{2}
$$

где $R_{1}=\left\langle x_{1}, \ldots, x_{n}\right\rangle$. Для любого элемента $p \in R_{1}$ через $p_{\alpha}$ будем обозначать коэффициент при $x_{\alpha}$ в $p$.

Покрасим все вершины дерева в два цвета правильным образом. Через $T_{0}$ и $T_{1}$ будем обозначать множества вершин цвета 0 и цвета 1 соответственно. Для $p \in R_{1}$ положим

$$
\widetilde{p}=\sum_{\alpha \in T_{0}} p_{\alpha} x_{\alpha}-\sum_{\beta \in T_{1}} p_{\beta} x_{\beta} .
$$

Лемма 5. Для любого $p \in R_{1}$ выполнено $р \widetilde{p}=0$. При этом $\operatorname{Ann}_{R_{1}} p=\langle\tilde{p}\rangle$ в том и только том случае, когда коэффициент $p_{\alpha}$ для всякой внутренней вериины $\alpha$ отличен от нуля.

ДоказАтельство. Пусть $q \in R_{1}$ таково, что $p q=0$. Рассмотрим $(\alpha, \beta) \in T$, причем $\alpha \in T_{0}$ и $\beta \in T_{1}$. Коэффициент при $x_{\alpha} x_{\beta}$ в произведении $p q$ равен $p_{\alpha} q_{\beta}+p_{\beta} q_{\alpha}=0$. Это означает, что векторы $\left(p_{\alpha},-p_{\beta}\right)$ и $\left(q_{\alpha}, q_{\beta}\right)$ пропорциональны. Если для каждой внутренней вершины $\alpha$ число $p_{\alpha}$ отлично от нуля, то коэффициенты пропорциональности на инцидентных ребрах равны. В силу связности дерева равны коэффициенты пропорциональности на всех ребрах, т.е. $q$ пропорционально $\widetilde{p}$.

Обратно, пусть $\alpha$ - внутренняя вершина, для которой $p_{\alpha}=0$. Рассмотрим представление $T=S_{1} \cup S_{2}$, где $S_{1}$ и $S_{2}$ - поддеревья и $S_{1} \cap S_{2}=\{\alpha\}$. Так как $\alpha$ внутренняя, можно считать, что в каждом из $S_{1}$ и $S_{2}$ больше одной вершины. Пусть $p^{1}$ и $p^{2}$ - ограничения $p$ на $S_{1}$ и $S_{2}$ соответственно. Заметим, что $p^{1}, p^{2} \in$ $\operatorname{Ann}_{R_{1}} p$, т.е. в случае $p^{1} \neq 0$ и $p^{2} \neq 0$ аннулятор $p$ не одномерен. Если $p^{i}$ равно нулю, то для любой вершины $\beta \in S_{i} \backslash\{\alpha\}$ выполнено $x_{\beta} \in \operatorname{Ann}_{R_{1}} p$, откуда следует, что аннулятор $p$ также не одномерен.

Из предложения 3 и лемм 5 и 4 получаем

ПредлОЖениЕ 4. Над алгеброй графа существуют несвободнъе модули нулевой G-размерности тогда и только тогда, когда граф является деревом. Множество классов изоморфизма несвободных ииклических модулей нулевой G-размерности над алгеброй дерева параметризуется элементами множества $P R_{1} \backslash H$, где $H$ - обгединение гиперплоскостей $\left\{p_{\alpha}=0\right\}$ по всем внутренним вершинам $\alpha$.

ДокАЗАТЕЛЬСтво. Положим $p=\sum_{i=1}^{n} x_{i}$ и $q=\widetilde{p}$. По лемме $5 p q=0$. Чтобы применить предложение 3 , осталось проверить, что $\operatorname{Ann}(p)=(q)$ и $\operatorname{Ann}(q)=(p)$. Действительно, из леммы 5 следует, что ядро умножения на $p$ $\left(\right.$ соответственно на $q$ ) как отображения $R_{1} \rightarrow R_{2}$ одномерно. Так как $\operatorname{dim} R_{1}=$ $\operatorname{dim} R_{2}+1$, это отображение сюръективно.

Обратно, пусть $\alpha$ - внутренняя вершина и $p_{\alpha}=0$. Так как $\operatorname{dim}_{k} \operatorname{Ann}_{R_{1}} p \geqslant 2$, размерности компонент модуля $R /(p)$ не будут удовлетворять условию 5$)$ из теоремы 1 .

То, что построенные для различных элементов $P R_{1} \backslash H$ модули не изоморфны, следует, например, из того, что у них разные аннуляторы. 
Далее мы обобщаем предложение 4 на все конечно порожденные неразложимые модули нулевой G-размерности. Каждый такой модуль $M$ порождается столбцами матрицы $A \in \operatorname{Mat}_{s}\left(R_{1}\right), A=\left(a_{i j}\right)_{i j=1}^{s}$. Через $A_{\alpha}$ будем обозначать матрицу $\left(\left(a_{i j}\right)_{\alpha}\right)_{i j=1}^{s}$. Матрицу $A \in \operatorname{Mat}_{s}\left(R_{1}\right)$, для которой для каждой внутренней вершины $\alpha$ дерева $T$ матрица $A_{\alpha}$ над полем $k$ невырожденная, будет называть невырожденной.

ТеОрема 2. $R$-модуль $M \subset R^{s}$, порожденный столбцами матрицы $A \in$ $\operatorname{Mat}_{s}\left(R_{1}\right)$, имеет нулевую $\mathrm{G}$-размерность тогда и только тогда, когда матрица $A$ невырожденная.

ДокАЗАТЕЛЬСтво. Зафиксируем невырожденную матрицу $A$.

Лемма 6. Пространство соотношений для системы столбцов А с коэффичиентами из $R_{1}$ имеет размерность $s$ над $k$. И если столбиъ матриць $B \in \operatorname{Mat}_{s}\left(R_{1}\right)$ образуют базис этого пространства соотношений, то матрица В также не вырождена.

ДокАЗАтЕЛьство. Будем строить столбец $v \in R_{1}^{s}$ такой, что $A v=0$. Это матричное равенство равносильно следующей системе, в которой $(\alpha, \beta)$ пробегает все ребра $T$ :

$$
A_{\beta} v_{\alpha}+A_{\alpha} v_{\beta}=0
$$

Если $\alpha$ - внутренняя вершина, то вектор $v_{\beta}$ из этого равенства определяется однозначно. Рассмотрим некоторую внутреннюю вершину $\alpha_{0}$. Тогда по $v_{\alpha_{0}}$ весь $v$ строится однозначно, откуда и следует $s$-мерность пространства соотношений.

Аналогичным образом, если столбцы матрицы $B \in \operatorname{Mat}_{s}\left(R_{1}\right)$ образуют базис пространства соотношений, то ее можно искать из начального условия $B_{\alpha_{0}}=E$ и соотношений $A_{\alpha} B_{\beta}+A_{\beta} B_{\alpha}=0$, где $(\alpha, \beta)$ пробегает все ребра дерева $T$. Таким образом, для всякой внутренней вершины $\alpha$ матрица $B_{\alpha}$ есть произведение в некотором порядке некоторых из матриц $A_{\beta}$ (где $\beta$ также внутренняя), им обратных, и матрицы $B_{\alpha_{0}}$, т.е. является невырожденной матрицей.

Следующее следствие вытекает из того, что условие на невырожденность матрицы $A$ сохраняется при транспонировании.

СлеДСтвиЕ 3. Лемма 6 остается справедливой при замене столбцов на строки.

Из леммы 6 мы получаем, что модуль $M$, задаваемый невырожденной матрицей $A$, обладает минимальной свободной резольвентой

$$
\cdots \rightarrow F_{2} \stackrel{\varphi_{2}}{\longrightarrow} F_{1} \stackrel{\varphi_{1}}{\longrightarrow} F_{0} \rightarrow M \rightarrow 0
$$

в которой для всех $i$ выполнено $F_{i} \cong R^{s}$ и $\varphi_{i}$ задается невырожденной матрицей $A_{i} \in \operatorname{Mat}_{s}\left(R_{1}\right)$.

Из леммы 1 следует, что сопряженный комплекс $0 \rightarrow M^{*} \rightarrow F_{0}^{*} \stackrel{\varphi_{1}^{*}}{\longrightarrow} F_{1}^{*} \rightarrow \cdots$ является ациклическим, так как все $\varphi_{i}^{*}$ задаются невырожденными матрицами $A_{i}^{T}$ и, следовательно, $\operatorname{dim}_{k} \operatorname{Ker} \varphi_{i}^{*}=s$ для всех $i$. Применяя лемму 6 к модулю $M^{*}$, заданному невырожденной матрицей $A^{T}$, получаем бесконечную в обе стороны свободную резольвенту для $M$. Снова применяя лемму 1 , получаем, 
что сопряженная к этой резольвенте последовательность также точна. Отсюда следует, что $M^{*}$ является горенштейново проективным, а значит, имеет нулевую G-размерность. Согласно замечанию $4 M$ также имеет нулевую G-размерность.

Пусть теперь модуль $M$, порожденный столбцами матрицы $A$, имеет нулевую G-размерность. Покажем, что $A$ невырождена. Обозначим через $B \in \operatorname{Mat}_{s}\left(R_{1}\right)$ матрицу, составленную из порождающих модуля сизигий.

Лемма 7. Пусть для некоторой внутренней вериины $\alpha_{0}$ дерева $T$ матрииа $A_{\alpha_{0}}$ вырождена. Тогда для любой вершины $\beta \in T$ матрица $B_{\beta}$ вырождена.

ДокАЗАТЕЛьство. Подвесим дерево $T$ за вершину $\alpha_{0}$. Пусть $\alpha_{1}$ и $\alpha_{2}$ смежны с $\alpha_{0}$. Через $T_{1}$ (соответственно $T_{2}$ ) обозначим максимальное поддерево, содержащее $\alpha_{1}$ (соответственно $\alpha_{2}$ ), в котором вершина $\alpha_{0}$ является висячей. Очевидно, $\alpha_{1} \notin T_{2}, \alpha_{2} \notin T_{1}$ и $T_{1} \cap T_{2}=\left\{\alpha_{0}\right\}$.

Через $\beta_{1}$ обозначим самую низкую (относительно исходного подвешивания) внутреннюю вершину $T$, лежащую в $T_{1}$, такую, что $A_{\beta_{1}}$ вырождена. $\mathrm{K} \beta_{1}$ подвешено несколько поддеревьев в $T_{1}$, обозначим их через $t_{1}, \ldots, t_{k}$, в которых матрицы всех внутренних вершин уже не вырождены. Обозначим сыновей вершины $\beta_{1}$ через $\gamma_{1}, \ldots, \gamma_{k}$ так, что $\gamma_{i} \in t_{i}$.

Построим ненулевой вектор $v \in R_{1}^{s}$ такой, что $A v=0$ и $v_{\alpha}=0$ при $\alpha=\beta_{1}$ и $\alpha \notin t_{1} \cup \cdots \cup t_{k}$. Для этого достаточно взять в качестве $v_{\gamma_{i}}$ произвольные ненулевые векторы из ядра $A_{\beta_{1}}$. Из доказательства леммы 6 следует, что $v$ определяется на остальных вершинах из $t_{1}, \ldots, t_{k}$ корректно и однозначно. Нетрудно видеть, что построенный вектор удовлетворяет соотношениям (1).

Так как $v$ является $k$-линейной комбинацией столбцов $B$, то для всех вершин $\alpha \notin t_{1} \cup \cdots \cup t_{k}$ матрица $B_{\alpha}$ вырождена. Проделав аналогичную операцию для $T_{2}$, получаем требуемое.

Теперь, перейдя при необходимости к модулю сизигий, допускаем, что все матрицы $A_{\alpha}$ вырождены.

Рассмотрим какие-либо две вершины $\beta_{1}$ и $\beta_{2}$, которые смежны хотя бы с одной висячей, или одну вершину $\beta_{1}$, которая смежна хотя бы с двумя висячими. Обозначим через $r$ максимум рангов $A_{\beta_{1}}$ и $A_{\beta_{2}}$ (или просто ранг $A_{\beta_{1}}$ в случае одной вершины), $r<s$. Так же, как и выше, можно построить $2(s-r)$ линейно независимых над $k$ элементов $v$ степени 1 из модуля сизигий, для которых $v_{\alpha}=0$ для всех вершин, за исключением висячих вершин, смежных с $\beta_{i}$. Если $2 r<s$, то это противоречит тому, что для всех модулей сизигий нашего модуля размерность первой градуированной компоненты равна $s$. Если $2 r \geqslant s$, то для матрицы $B$, задающей модуль сизигий, получим, что

$$
\operatorname{rank} B_{\beta_{i}} \leqslant s-(2 s-2 r)=2 r-s<r .
$$

Таким образом, после конечного числа шагов мы окажемся в ситуации $2 r<s$, невозможность которой доказана выше.

Теорема 2 доказана.

СЛЕДСТвИЕ 4. Классъ изоморфизмов модулей нулевой G-размерности с минимальным числом порождающих $b$, не содержащих свободных прямых слагаемых, над k-алгеброй дерева $T$ с $n$ вершинами находятся в биективном соответствии с классами относительного подобия наборов из $n-1$ матрии, 
размера $b \times b$ над $k$, занумерованных вершинами дерева, кроме одной фиксированной внутренней вершины, в которых матрицы, соответствующие внутренним вериинам дерева, являются невырожденными.

\section{§ 6. Неприводимые модули нулевой G-размерности над алгебрами деревьев}

Пусть $R$ - локальная или стандартная градуированная алгебра с максимальным или максимальным однородным идеалом $\mathfrak{m}$, причем $\operatorname{depth} R=0$. Рассмотрим $R$-модуль $M$ нулевой $\mathrm{G}$-размерности, не содержащий свободных подмодулей. Такой модуль будем называть приводимым, если он содержит собственный подмодуль $N$ нулевой G-размерности (равносильно: собственный подмодуль $N$, для которого $M / N$ имеет нулевую G-размерность).

Лемма 8. Модуль $M$ приводим тогда и только тогда, когда $M^{*}$ приводим.

Доказательство. Пусть $0 \rightarrow N \rightarrow M \rightarrow P \rightarrow 0$, где $N$ и $P$ имеют нулевую G-размерность. Тогда $0 \rightarrow P^{*} \rightarrow M^{*} \rightarrow N^{*} \rightarrow 0$ также точна, т.е. $M^{*}$ приводим. Обратное верно, так как $M=M^{* *}$.

Лемма 9. Пусть $R$ - градуированная алгебра и $M=M_{1} \oplus M_{2}$ - градуированный модуль с двумя ненулевыми компонентами. Тогда всякий подмодуль $N \subset M$ изоморфен однородному подмодулю.

ДокАЗАТЕЛЬство. Пусть $a_{1}, \ldots, a_{q}$ - минимальная система порождающих модуля $N$, причем $a_{i}=a_{i}^{\prime}+a_{i}^{\prime \prime}$, где $a_{i}^{\prime} \in M_{1}$ и $a_{i}^{\prime \prime} \in M_{2}$. После $k$-линейной замены можно считать, что $a_{1}^{\prime}, \ldots, a_{p}^{\prime}$ линейно независимы над $k$, а $a_{p+1}^{\prime}=\cdots=$ $a_{q}^{\prime}=0$. Тогда набор $a_{1}^{\prime}, \ldots, a_{p}^{\prime}, a_{p+1}^{\prime \prime}, \ldots, a_{q}^{\prime \prime}$ является минимальной системой порождающих однородного подмодуля $N^{\prime}$. Имеют место представления

$$
\begin{aligned}
N & =\left\langle a_{1}, \ldots, a_{p}\right\rangle \oplus\left\langle a_{p+1}^{\prime \prime}, \ldots, a_{q}^{\prime \prime}\right\rangle, \\
N^{\prime} & =\left\langle a_{1}^{\prime}, \ldots, a_{p}^{\prime}\right\rangle \oplus\left\langle a_{p+1}^{\prime \prime}, \ldots, a_{q}^{\prime \prime}\right\rangle .
\end{aligned}
$$

Отображение $\varphi:\left\langle a_{1}, \ldots, a_{p}\right\rangle \rightarrow\left\langle a_{1}^{\prime}, \ldots, a_{p}^{\prime}\right\rangle$, заданное формулой

$$
\varphi\left(r_{1} a_{1}+\cdots+r_{p} a_{p}\right)=r_{1} a_{1}^{\prime}+\cdots+r_{p} a_{p}^{\prime}
$$

задает изоморфизм между $N^{\prime}$ и $N$.

Далее предполагаем, что $\mathfrak{m}^{3}=0$, т.е. можно рассматривать $R$ как градуированную алгебру, а модули нулевой G-размерности - как градуированные модули.

Лемма 10. Модуль $М$ приводим тогда и толъко тогда, когда его модуль сизигий приводим.

ДокАЗАТЕЛЬСтво. Пусть $M$ приводим и содержит однородный собственный модуль $N \subset M$ нулевой G-размерности. Минимальная система порождающих для $N$ является частью минимальной системы порождающих для $M$, т.е. $\operatorname{Syz}(N)$ естественным образом вкладывается в $\operatorname{Syz}(M)$. Так как $M$ не содержит свободных подмодулей, $\operatorname{Syz}(N)$ является собственным подмодулем $\operatorname{Syz}(M)$. 
Обратно, пусть модуль сизигий $P=\operatorname{Syz}(M)$ приводим. Имеет место точная последовательность

$$
0 \rightarrow P \rightarrow R^{b} \rightarrow M \rightarrow 0,
$$

где $b=\operatorname{dim}_{k} M_{1}$. Тогда точна и последовательность $0 \rightarrow M^{*} \rightarrow R^{b} \rightarrow P^{*} \rightarrow 0$, откуда Syz $P^{*}=M^{*}$. Модуль $P$ приводим, значит, $P^{*}$ приводим, а значит, $M^{*}=\operatorname{Syz} P^{*}$ приводим, откуда уже и $M$ приводим.

Будем называть матрицу $A \in \operatorname{Mat}_{b}\left(R_{1}\right)$ приводимой, если существуют такие обратимые матрицы $C, D \in \operatorname{Mat}_{b}(k)$ и такое натуральное $l, 0<l<b$, что матрица $C A D$ имеет угол нулей размера $(b-l) \times l$.

Лемма 11. Пусть $M=\operatorname{Im} f-$ приводимый модуль нулевой G-размерности с минимальной системой порождающих из $b$ элементов, где $\varphi: R^{b} \rightarrow R^{b}$ задается матрицей $A \in \operatorname{Mat}_{b}\left(R_{1}\right)$. Тогда матрица $A$ приводима.

ДоказАтельство. Модуль $M$ изоморфен $\operatorname{Syz}(P)$ для некоторого $P$, содержащего подмодуль $N$ с минимальной системой из $l$ порождающих. Из доказательства леммы 10 видно, что при естественном вложении $\operatorname{Syz}(P)$ в $R^{b}$ модуль $\operatorname{Syz}(P)$ содержит подмодуль с $l$ порождающими, содержащийся в подмодуле, порожденном первыми $l$ базисными элементами в $R^{b}$. Иначе говоря, $M=\operatorname{Syz}(P)$ задается матрицей $B \in \operatorname{Mat}_{b}\left(R_{1}\right)$ с углом нулей размера $(b-l) \times l$. Значит, существуют матрицы $C, D \in \mathrm{GL}_{b}(k)$ такие, что $B=C A D$.

Теорема 3. Пусть $R$ - алгебра дерева и $M$-модуль нулевой G-размернос-

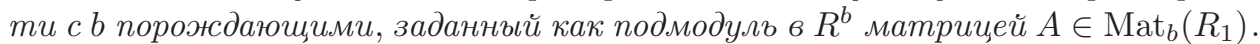
Тогда $M$ приводим, если и только если существуют матриць $C, D \in \mathrm{GL}_{b}(k) u$ число $0<l<b$ такие, что матрииа $C A D$ имеет угол нулей размера $(b-l) \times l$.

ДокАзАтЕльство. В одну сторону теорема следует из леммы 11. Если такие $C$ и $D$ существуют, то будем предполагать, что $A$ уже имеет угол нулей размера $(b-l) \times l$. Через $B$ обозначим левую верхнюю подматрицу размера $l \times l$. Из невырожденности $A$ следует невырожденность $B$, а значит, по теореме 2 столбцы матрицы $B$ порождают модуль нулевой G-размерности.

ТЕОрема 4. Для всякого b над алгеброй дерева существует неприводимый модуль нулевой $\mathrm{G}-$ размерности с $b$ порождающими.

ДокАЗАТЕЛЬство. Если $b=1$, то всякий несвободный циклический модуль нулевой G-размерности неприводим.

Пусть $b \geqslant 2$. Выберем в дереве внутреннюю вершину $\alpha$ и две произвольные вершины $\beta_{1}$ и $\beta_{2}$. Для всех остальных вершин $\gamma$ выбираем такие матрицы $A_{\gamma}$ с единственным ограничением, что для внутренних вершин $\gamma$ матрица $A_{\gamma}$ невырожденная. Полагаем $A_{\alpha}=E$, а матрицы $A_{\beta_{1}}$ и $A_{\beta_{2}}$ выбираем такими, что для любых $C, D \in \mathrm{GL}_{b}(k)$ матрицы $C D, C A_{\beta_{1}} D$ и $C A_{\beta_{2}} D$ не имеют общего инвариантного подпространства $L \subset k^{b}$. Если бы такие $C, D$ и $L$ существовали, то

$$
D L \subset C^{-1} L, \quad A_{\beta_{i}}(D L) \subset C^{-1} L, \quad i=1,2 .
$$

Так как $\operatorname{dim} D L=\operatorname{dim} C^{-1} L$, то $D L=C^{-1} L$, и $A_{\beta_{1}}$ и $A_{\beta_{2}}$ имели бы общее инвариантное подпространство. Чтобы такого не произошло, достаточно взять в качестве $A_{\beta_{1}}$ жорданову клетку с ненулевым собственным значением, а в качестве $A_{\beta_{2}}$ - транспонированную жорданову клетку.

Несложно видеть, что построенная матрица $A$ будет неприводимой. 
Теорема 5. Пусть поле $k$ не является алгебраическим расширением конечного поля и $R$ - алгебра дерева, не являющегося звездой, над $k$. Тогда для любого $b \geqslant 2$ существует неприводимый модуль с $b$ образующими, обладающий непериодической резольвентой.

ДоказАТЕльство. Так как дерево не является звездой, в нем имеется простой путь длины 4, вершины которого мы обозначим через 1, 2, 3 и 4. Выберем невырожденную матрицу $A \in \operatorname{Mat}_{b}\left(R_{1}\right)$, для которой $A_{2}=E, A_{1}$ - жорданова клетка, $A_{4}=A_{1}^{T}$ и $A_{3}=\operatorname{diag}(1, \ldots, 1, a)$, где $a$ не является корнем из 1. Пусть $M$ - модуль, задаваемый матрицей $A$, и $M^{(i)}$ обозначает его $i$-й модуль сизигий, заданный матрицей $A^{(i)}$. Покажем, что $M^{(i)}$ не изоморфен $M$.

Предположим, что $M$ изоморфен $M^{(i)}$. Пользуясь соотношениями из леммы 6, несложно проверить по индукции, что

$$
A_{2}^{(i)}=E, \quad A_{1}^{(i)}=(-1)^{i} A_{1}, \quad A_{3}^{(i)}=(-1)^{i} A_{3}, \quad A_{4}^{(i)}=A_{3}^{-i} A_{4} A_{3}^{i} .
$$

Если $M \cong M^{(i)}$, то $M \cong M^{(2 i)}$, поэтому можно считать $i$ четным. В случае такого изоморфизма нашлись бы матрицы $U$ и $V$ такие, что

$$
U V=E, \quad U A_{1} V=A_{1}, \quad U A_{3} V=A_{3}, \quad A_{4}^{(i)}=U A_{4} V .
$$

Тогда $V=U^{-1}, U$ перестановочна с $A_{1}$ и $A_{3}$, а значит, является скалярной. Тогда $A_{4}^{(i)}=A_{4}$, но ни одна степень матрицы $A_{3}$ не перестановочна с жордановой клеткой.

Аналогично, $M^{(i)}$ не изоморфно $M^{(j)}$ при $i \neq j$, откуда и следует, что резольвента непериодична.

ЗАмЕчАниЕ 6 . Если $k$ является алгебраическим расширением конечного поля, то модуль $M$ определен над некоторым конечным подполем $k$. Более того, все модули $M^{(i)}$ определены над этим же конечным подполем $k$ и имеют то же число порождающих. Таких модулей с точностью до изоморфизма лишь конечное число, а значит, резольвента заведомо будет периодической.

\section{§ 7. Неразложимые модули нулевой G-размерности над алгебрами деревьев}

В этом параграфе мы получим результаты, аналогичные результатам из $\S 6$ для неразложимых модулей нулевой G-размерности. Пусть $R$ - локальная или стандартная градуированная алгебра с максимальным или максимальным однородным идеалом $\mathfrak{m}$, причем $\operatorname{depth} R=0$. Модуль $M$ нулевой G-размерности над алгеброй $R$ будет называть неразложимьм, если он не раскладывается в прямую сумму ненулевых подмодулей. Заметим, что в силу предложения 1 в градуированном случае это равносильно тому, что он не раскладывается в прямую сумму ненулевых однородных подмодулей.

ЛЕмма 12. 1. Если $M$ разложим в прямую сумму $K \oplus L$, то $K$ u $L$ имеют нулевую $\mathrm{G}-$ размерность.

2. $M$ неразложим тогда и только тогда, когда $M^{*}$ неразложим.

3. $M$ неразложим тогда и только тогда, когда $\operatorname{Syz}(M)$ неразложим. 
ДоказАтельство. Пусть $M=K \oplus L$. Тогда

$$
M^{*}=K^{*} \oplus L^{*}, \quad M \cong M^{* *}=K^{* *} \oplus L^{* *},
$$

а значит, из разложимости $M$ следует разложимость $M^{*}$ и наоборот. Из инъективности и сюръективности $M \rightarrow M^{* *}$ следует инъективность и сюръективность $K \rightarrow K^{* *}$ и $L \rightarrow L^{* *}$. Так как Ext коммутирует с прямыми суммами, то отсюда следует, что $K$ и $L$ имеют нулевую G-размерность.

Из разложимости $M$ следует разложимость $P=\operatorname{Syz}(M)$. Покажем обратную импликацию. Аналогично доказательству леммы $10 \mathrm{Syz} P^{*}=M^{*}$. Если $P$ разложим, то $P^{*}$ разложим, т.е. Syz $P^{*}=M^{*}$ разложим, а значит, и $M$ разложим.

Пусть теперь $R$ - алгебра дерева. Матрицу $A \in \operatorname{Mat}_{b}\left(R_{1}\right)$ будем называть разложимой, если существуют такие обратимые матрицы $C, D \in \operatorname{Mat}_{b}(k)$, что матрица $C A D$ имеет блочно-диагональный вид.

ПРеДЛОЖЕНИЕ 5. Пустъ $R$ - алгебра дерева, $M-R$-модулъ с $b$ порожда-

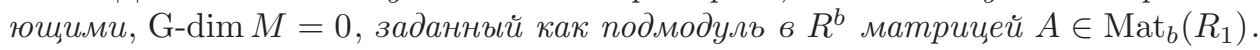
В этом случае $M$ разложим тогда и только тогда, когда $A$ разложима.

ДоказАтельство. В случае разложимости $A$ разложимость $M$ очевидна.

Пусть $M$ разложим. Тогда разложим также модуль $N=(\operatorname{Syz} M)^{*}$. Пусть $N=K \oplus L, f_{1}, \ldots, f_{l}$ - базис в $K$, а $e_{1}, \ldots, e_{b-l}-$ базис в $L$. В базисе $f_{1}, \ldots, f_{l}$, $e_{1}, \ldots, e_{b-l} \operatorname{Syz}(N)$ будет задан блочно-диагональной матрицей с двумя блоками размеров $l \times l$ и $(b-l) \times(b-l)$. Так как $\operatorname{Syz}(N)=M^{*}$, то $M$ тоже будет задан в соответствующем базисе блочно-диагональной матрицей, что и требовалось.

\section{§ 8. CI-размерность модулей над алгебрами деревьев}

Наряду с G-размерностью была введена серия гомологических размерностей модулей, совпадающих с проективной размерностью, когда последняя конечна, и совпадающих между собой, когда какие-либо из них конечны. Мы рассмотрим здесь для модулей над алгебрами деревьев CI-размерность, введенную Л. Л. Аврамовым, В. Н. Гашаровым и И. В. Пеевой (см. [16]).

ОПРЕДЕЛЕНИЕ 6. Деформацией кольца $R$ называется сюръективный гомоморфизм локальных колец $Q \rightarrow R$, ядро которого порождено регулярной последовательностью.

Квазидеформацией кольца $R$ называется диаграмма гомоморфизмов локальных колец $R \rightarrow R^{\prime} \leftarrow Q$, где $R \rightarrow R^{\prime}$ - плоское расширение, а $Q \rightarrow R^{\prime}-$ деформация.

Говорят, что $R$-модуль $M$ имеет конечную CI-размерность, если для некоторой квазидеформации кольца $R$ модуль $R^{\prime} \otimes_{R} M$ имеет конечную проективную размерность над $Q$.

Модуль конечной CI-размерности имеет конечную G-размерность, но обратное, вообще говоря, неверно. Над алгебрами Ёсино, полученными при помощи деформации, все модули нулевой G-размерности имеют нулевую CI-размерность. 
Теорема 6. Над алгеброй дерева, не являющегося звездой, все несвободнъе модули имеют бесконечную СІ-размерность.

ДокАзАтЕльство. Для доказательства используется техника, развитая в работах [17]-[19]. Пусть $R$ - стандартная градуированная алгебра над полем $k$ с квадратичными соотношениями, т.е. факторалгебра свободной ассоциативной (тензорной) алгебры $T\left(R_{1}\right)$ по идеалу, порожденному некоторым подпространством $Q \subset R_{1} \otimes_{k} R_{1}$. Тогда двойственная к ней алгебра $T\left(R_{1}^{*}\right) /\left(Q^{\perp}\right)$, где $Q^{\perp} \subset R_{1}^{*} \otimes R_{1}^{*}$ - ортогональное дополнение к $Q$, изоморфна подалгебре в алгебре Йонеды $\operatorname{Ext}_{R}^{*}(k, k)$, порожденной $\operatorname{Ext}_{R}^{1}(k, k)$. В случае, когда $R$ козюлева, $\operatorname{Ext}_{R}^{1}(k, k)$ порождает всю алгебру $\operatorname{Ext}_{R}^{*}(k, k)$. Алгебра $\operatorname{Ext}^{*}(k, k)$ является универсальной обертывающей некоторой градуированной алгебры Ли гомотопической алгебры Ли $\pi^{*}(R)$ алгебры $R$. Согласно [16], [18], если $M-$ модуль конечной CI-размерности, то левый $\operatorname{Ext}_{R}^{*}(k, k)$-модуль $\operatorname{Ext}_{R}^{*}(M, k)$ конечно порожден над $k$-подалгеброй в $\operatorname{Ext}_{R}^{*}(k, k)$, порожденной центральными элементами степени 2. Поэтому если таких элементов нет, то пространство $\operatorname{Ext}_{R}^{*}(k, k)$ должно быть конечномерно над $k$, что означало бы, что модуль $M$ имеет конечную проективную размерность. В нашем случае она была бы равна нулю, т.е. модуль $M$ был бы свободен. Поэтому достаточно доказать, что $\pi^{2}(R)$ не содержит центральных элементов степени 2.

Доказывать это будем, выписав явно определяющие соотношения для $\operatorname{Ext}_{R}^{2}(k, k)$ и вычислив базис Грёбнера для идеала, ими порожденного. Так как $R$ задается в свободной ассоциативной алгебре соотношениями второй степени, алгебра $\operatorname{Ext}_{R}^{*}(k, k)$ порождается элементами первой степени (см. [19; теорема 1.2]). Эти соотношения второй степени можно выписать явно: $R=$ $k\left\langle x_{1}, \ldots, x_{n}\right\rangle / W$, где $W \subset V \otimes V, V=\left\langle x_{1}, \ldots, x_{n}\right\rangle$, - подпространство, натянутое на соотношения вида $x_{i} x_{j}-x_{j} x_{i}$ для всех $i$ и $j$ и $x_{i} x_{j}$ при $(i, j) \notin T$. Так как размерность пространства $V \otimes V$ равна $n^{2}$, двойственное подпространство $W^{\perp}$ в $V^{*} \otimes V^{*}$ имеет размерность $n-1$. Если обозначить базис в $V^{*}$, двойственный к $x_{1}, \ldots, x_{n}$, через $y_{1}, \ldots, y_{n}$, то базис этого двойственного подпространства легко выписать: это все выражения вида $y_{i} y_{j}+y_{j} y_{i}$ при $(i, j) \in T$.

Согласно [19; следствие 1.3]

$$
\operatorname{Ext}_{R}^{*}(k, k)=k\left\langle y_{1}, \ldots, y_{n}\right\rangle /\left(W^{\perp}\right)
$$

имеет стандартную градуировку. Для вычислений в этой алгебре будем использовать базисы Грёбнера. Введем на мономах степенное лексикографическое упорядочение: будем сравнивать сначала степени мономов, а в случае их равенства сами мономы сравниваем лексикографически. Порядок на переменных: $y_{1}>y_{2}>\cdots>y_{n}$. Для набора порождающих

$$
\left\{f_{i j}=y_{i} y_{j}+y_{j} y_{i} \mid(i, j) \in T\right\}
$$

применим алгоритм Бухбергера. Будет построен, вообще говоря, бесконечный набор многочленов. Однако так как исходные многочлены были однородны, построенный набор будет также состоять из однородных многочленов. Нас интересуют в нем многочлены степени не больше 3.

Лемма 13. В построенном базисе Грёбнера многочлены второй степени исчерпываются многочленами $f_{i j}$, а многочлены третъей степени таковы:

$$
\left\{y_{i} y_{k} y_{j}-y_{j} y_{i} y_{k} \mid i<j<k,(i, j),(j, k) \in T\right\} .
$$


ДокАЗАтЕльство. $S$-полином, взятый от однородных многочленов степеней $d_{1}$ и $d_{2}$, имеет степень не меньше $\max \left(d_{1}, d_{2}\right)+1$. Поэтому нам достаточно рассмотреть $S$-полиномы многочленов $f_{i j}$. Единственное возможное зацепление - зацепление между многочленами $f_{i j}$ и $f_{j k}$, где $i<j<k$. В таком случае $S$-полином имеет вид $f_{i j} y_{k}-y_{i} f_{j k}=y_{j} y_{i} y_{k}-y_{i} y_{k} y_{j}$. Старший моном в этом многочлене $y_{i} y_{k} y_{j}$ не редуцируется дальше, так как в дереве не могут быть одновременно ребра $(i, j),(j, k)$ и $(i, k)$. Дальнейший ход алгоритма Бухбергера будет только увеличивать степень. Таким образом, полученный набор многочленов исчерпывает элементы базиса Грёбнера до степени 3.

Рассмотрим некоторый элемент второй степени в $\operatorname{Ext}_{R}^{*}(k, k)$

$$
a=\sum_{(i, j) \notin T}\left(a_{i j}^{\prime} y_{i} y_{j}+a_{j i}^{\prime \prime} y_{j} y_{i}\right)+\sum_{(i, j) \in T, i<j} b_{i j} y_{j} y_{i} \text {. }
$$

Нас интересуют коммутаторы вида $\left[a, y_{k}\right]$. Так как редукция относительно нашего базиса Грёбнера заменяет моном на моном с тем же набором переменных, равенство этого коммутатора нулю равносильно равенству нулю коммутаторов $y_{k}$ с каждым из слагаемых из обеих сумм в формуле (2). Покажем, что для любого элемента $a$ найдется $k$ такой, что $\left[a, y_{k}\right] \neq 0$. Разберем несколько случаев.

1. Для некоторого $i$ моном $y_{i}^{2}$ входит в $a$ с ненулевым коэффициентом. Пусть $j$ - вершина, не смежная с $i$ (она найдется в силу условия на дерево $T$ ). Тогда $\left[y_{i}^{2}, y_{j}\right]=y_{i}^{2} y_{j}-y_{j} y_{i}^{2} \neq 0$, так как ни один из этих мономов не редуцируется при помощи базиса Грёбнера.

2. Для некоторых $i$ и $j$ таких, что $(i, j) \notin T$, один из мономов $y_{i} y_{j}$ и $y_{j} y_{i}$ входит в $a$ с ненулевым коэффициентом. Тогда коммутатор $\left[a_{i j}^{\prime} y_{i} y_{j}+a_{j i}^{\prime \prime} y_{j} y_{i}, y_{i}\right]$ имеет вид $\alpha y_{i}^{2} y_{j}+\beta y_{i} y_{j} y_{i}+\gamma y_{j} y_{i}^{2}$, причем хотя бы одно из чисел $\alpha$ и $\gamma$ не равно нулю. Это выражение не равно нулю, так как ни один из мономов не редуцируется при помощи базиса Грёбнера.

3. Для некоторых $i<j,(i, j) \in T$, моном $y_{j} y_{i}$ входит в $a$ с ненулевым коэффициентом. Пусть $k$-вершина, не смежная с $i$. Тогда рассмотрим коммутатор $\left[y_{j} y_{i}, y_{k}\right]=y_{j} y_{i} y_{k}-y_{k} y_{j} y_{i}$. Если $(j, k) \notin T$, то ни один из этих мономов не редуцируется, а значит, и весь коммутатор не равен нулю. Если же $(j, k) \in T$, то возможны два случая:

(a) $j<k$; тогда старшим мономом в коммутаторе является $y_{j} y_{i} y_{k}$, который не редуцируется;

(b) $j>k$; тогда возможна редукция при помощи $f_{k j}: y_{j} y_{i} y_{k}-y_{k} y_{j} y_{i} \rightarrow$ $y_{j} y_{i} y_{k}+y_{j} y_{k} y_{i}$, и в полученном многочлене ни один моном не редуцируется.

Таким образом, в $\operatorname{Ext}_{R}^{*}(k, k)$ нет центральных элементов второго порядка, что и требовалось доказать.

ЗАмечАниЕ 7. Доказать отсутствие нетривиальных деформаций у алгебры дерева, отличного от звезды, в случае бесконечного поля $k$ можно и элементарными методами.

Действительно, ряд Гильберта для $R$ имеет вид

$$
H_{R}(t)=1+n t+(n-1) t^{2} .
$$


При факторизации по неделителю нуля степени $d$ ряд Гильберта умножается на $\left(1-t^{d_{i}}\right)$. Таким образом, ряд для деформации должен иметь вид

$$
H_{S}(t)=\frac{H_{A(T)}(t)}{\prod\left(1-t^{d_{i}}\right)} .
$$

Над бесконечным полем можно считать, что порождающие имеют степень 1 , а значит, ряд $H_{S}(t)$ переписывается в виде

$$
\frac{p(t)}{(1-t)^{k}} .
$$

Так как $d_{i}>1$, то получаем, что $k=1$ и $p(t)=1+(n-1) t$. Поэтому если деформация возможна, то она осуществляется одним регулярным элементом степени 2.

Пусть такая деформация существует. Рассмотрим идеал

$$
I=\left(x_{1}^{2}, \ldots, x_{n}^{2}, x_{i} x_{j} \mid(i, j) \notin T\right) .
$$

Очевидно,

$$
R=k\left[x_{1}, \ldots, x_{n}\right] / I .
$$

Пусть тогда деформация $S$ имеет вид $k\left[x_{1}, \ldots, x_{n}\right] / J$, где $J \subset I$. Размерности компонент степени 2 идеалов $I, J$ отличаются на единицу: $\operatorname{dim} I_{2}=\operatorname{dim} J_{2}+1$. Тогда для любого $y \in I_{2} \backslash J_{2}$ образ $\bar{y}$ в $S=k\left[x_{1}, \ldots, x_{n}\right] / J$ должен быть делителем нуля. Разберем два случая.

Случай 1: $y$ может быть выбран в виде $x_{i} x_{j}$ с $i \neq j$. Рассмотрим размерность $\operatorname{dim}\left(\bar{y}\left\langle x_{1}, \ldots, x_{n}\right\rangle\right)$. Так как $\bar{y}$ не является делителем нуля, она должна быть равна $n$. Рассмотрим некоторое $k$. Тогда:

1) среди пар $i, k$ и $j, k$ есть либо пара равных, либо пара различных несмежных вершин; тогда соответствующее произведение по модулю $J_{2}$ пропорционально $x_{i} x_{j}$, а весь моном $x_{i} x_{j} x_{k}$ пропорционален $x_{i}^{2} x_{j}$ или $x_{i} x_{j}^{2}$;

2) $(i, k) \in T$ и $(j, k) \in T$; таких $k$ не более одного. (Действительно, если бы нашлись еще $k^{\prime}$ с таким свойством, в дереве $T$ был бы цикл $i, k, j, k^{\prime}$.)

Таким образом, рассматриваемое пространство имеет размерность не более 3. При $n \leqslant 3$ все деревья являются звездами.

Случай 2: все $x_{i} x_{j}$ с $i \neq j$ и $(i, j) \notin T$ лежат в $J$, а $y$ можно выбрать равным $x_{i}^{2}$. Снова рассмотрим пространство $\bar{y}\left\langle x_{1}, \ldots, x_{n}\right\rangle$. Пусть степень вершины $x_{i}$ равна $d$. Пусть $(i, j) \notin T$; тогда $x_{i}^{2} x_{j}$ лежит в $J$, а значит, размерность рассматриваемого пространства не превосходит $d+1$. Это возможно только в случае $d=n-1$, что соответствует только звезде.

\section{Список литературы}

[1] Y. Yoshino, Cohen-Macaulay modules over Cohen-Macaulay rings, London Math. Soc. Lecture Note Ser., 146, Cambridge Univ. Press, Cambridge, 1990, viii+177 pp.

[2] R. Takahashi, "On the number of indecomposable totally reflexive modules", Bull. Lond. Math. Soc., 39:3 (2007), 487-492.

[3] R. Takahashi, "An uncountably infinite number of indecomposable totally reflexive modules", Nagoya Math. J., 187 (2007), 35-48. 
[4] L. W. Christensen, Gorenstein dimensions, Lecture Notes in Math., 1747, SpringerVerlag, Berlin, 2000, viii+204 pp.

[5] Y. Yoshino, "Modules of G-dimension zero over local rings with the cube of maximal ideal being zero", Commutative algebra, singularities and computer algebra (Sinaia, 2002), NATO Sci. Ser. II Math. Phys. Chem., 115, Kluwer Acad. Publ., Dordrecht, 2003, 255-273.

[6] R. Takahashi, "On the category of modules of Gorenstein dimension zero", Math. Z., 251:2 (2005), 249-256.

[7] R. Takahashi, "On the category of modules of Gorenstein dimension zero. II", J. Algebra, 278:1 (2004), 402-410.

[8] R. Takahashi, "Modules of G-dimension zero over local rings of depth two", Illinois J. Math., 48:3 (2004), 945-952.

[9] O. Veliche, "Construction of modules with finite homological dimensions", J. Algebra, 250:2 (2002), 427-449.

[10] A. Beilinson, V. Ginzburg, W. Soergel, "Koszul duality patterns in representation theory", J. Amer. Math. Soc, 9:2 (1996), 473-527.

[11] H. Holm, "Construction of totally reflexive modules from an exact pair of zero divisors", Bull. Lond. Math. Soc., 43:2 (2011), 278-288.

[12] O. Celikbas, M. Gheibi, R. Takahashi, "Brauer-Thrall for totally reflexive modules over local rings of higher dimension", Algebr. Represent. Theory, 17:3 (2014), 997-1008.

[13] L. W. Christensen, D. A. Jorgensen, H. Rahmati, J. Striuli, R. Wiegand, "BrauerThrall for totally reflexive modules", J. Algebra, 350:1 (2012), 340-373.

[14] H. Rahmati, J. Striuli, R. Wiegand, "A construction of totally reflexive modules", Algebr. Represent. Theory, 19:1 (2016), 103-111.

[15] R.P. Stanley, Combinatorics and commutative algebra, Progr. in Math., 41, Birkhäuser, Basel, 1996.

[16] L. L. Avramov, V. N. Gasharov, I. V. Peeva, "Complete intersection dimension", Inst. Hautes Études Sci. Publ. Math., 86 (1997), 67-114.

[17] L. L. Avramov, "Infinite free resolutions", Six lectures on commutative algebra (Bellaterra, 1996), Progr. Math., 166, Birkhäuser, Basel, 1998, 1-118.

[18] L. L. Avramov, Li-Chuan Sun, "Cohomology operators defined by a deformation", J. Algebra, 204:2 (1998), 684-710.

[19] C. Lofwall, "On the subalgebra generated by the one-dimensional elements in the Yoneda Ext-algebra", Algebra, algebraic topology and their interactions (Stockholm, 1983), Lecture Notes in Math., 1183, Springer-Verlag, Berlin, 1986, 291-338.

\section{Евгений Соломонович Голод}

(Evgueniy S. Golod)

Поступила в редакцию

Механико-математический факультет, 27.06 .2015

Московский государственный университет

имени М.В.Ломоносова

E-mail: golod@mech.math.msu.su

\section{Глеб Александрович Погудин}

\section{(Gleb A. Pogudin)}

Механико-математический факультет,

Московский государственный университет

имени М.В.Ломоносова

E-mail: pogudin.gleb@gmail.com 